www.nature.com/jhg

\title{
A commentary on assignment of Y-chromosomal SNPs found in Japanese population to Y-chromosomal haplogroup tree
}

\author{
Isao Yuasa
}

Journal of Human Genetics (2013) 58, 181; doi:10.1038/jhg.2013.11; published online 7 March 2013

$\mathrm{T}$

The binary polymorphisms on the human Y chromosome are useful uniparental markers not only for understanding human evolutionary histories but also for individualizing forensic biological materials. ${ }^{1,2}$ In 2008, a monophyletic phylogenetic tree of the $\mathrm{Y}$ chromosome was extensively revised, containing 311 distinct haplogroups based on approximately 600 binary markers. ${ }^{3}$ The haplogroups (branches) are defined by at least one binary polymorphism within the nonrecombining portion of the $\mathrm{Y}$ chromosome. New binary markers have been discovered in different populations, and have been assigned or re-assigned to upgrade the Y-chromosome haplogroup tree, improving the phylogenetic resolving power of the tree., ${ }^{4,5}$

In this issue of the journal, Naitoh et al. ${ }^{6}$ investigated 121 binary polymorphisms found in a Japanese population of the Japanese Single-Nucleotide Polymorphisms (JSNP) database and five new additional non-JSNP markers found through the analysis of PCR products. They determined the phylogenetic positions of 14 binary markers that define the new terminal branches and several markers, which are phylogenetically equivalent to previously reported mutations. They assigned five JSNP markers to the Y-chromosome tree in a previous study, ${ }^{7}$ and these markers were incorporated into the revised tree. ${ }^{3}$ The authors have provided us with the most detailed phylogenetic tree of Japanese Y chromosomes and frequency data on the $\mathrm{Y}$ haplogroups.

The three clades C, D and O comprise the majority of the Japanese. The C1-M105 and D2-M55 lineages, associated with Jomon

Dr I Yuasa is at Division of Legal Medicine, Faculty of Medicine, Tottori University, Yonago, Japan.

E-mail: yuasai@med.tottori-u.ac.jp
Y-chromosome lineages, are almost entirely restricted to the Japanese. The O2b-SRY465 lineages, associated with Yayoi lineages, are abundant in the Koreans and Japanese., ${ }^{8,9}$ Almost all binary markers were assigned to one of the three clades in their study. ${ }^{6}$ The $\mathrm{C}^{*}$-M217 paragroup was divided into three haplogroups by two mutations. The D2 $\mathrm{a}^{*}$-M116a paragroup (11.4\%) was also trifurcated, and the frequency of D2a*-M116a and the new lineage D2a-022456 was 5.3\%. The D2a1b-022457 lineage was subdivided, but the new haplogroup D2a1b-006841 was predominant. D2a1b-006841 and O2bSRY465 accounted for $19.8 \%$ and $33.5 \%$ of 263 investigated Japanese chromosomes, respectively. These are the reasons why the value of haplogroup diversity increased only slightly from $86.2 \%$ for 18 haplogroups $^{7}$ to $87.5 \%$ for $32 .{ }^{6}$ Although D2a1b-006841 and O2b-SRY465 are relatively young, ${ }^{8,9}$ further efforts are needed to subdivide them.

Further studies of East Asian populations are also needed to establish the value of the newly assigned markers. The two mutations defining haplogroups C3-64562+13 and C3-2613-27 would be useful for the investigation of populations with $\mathrm{C} 3^{\star}-\mathrm{M} 217$. The detailed distribution of D2a ${ }^{*}-$ M116a and D2a-022456 across the Japanese archipelago is important to clarify the diversity of the Jomon lineages and regional differences in the Japanese. In their study, ${ }^{6}$ the binary markers were investigated using single-strand conformation polymorphism technique, which is tedious, laborious and timeconsuming for routine analysis. A simple multiplex genotyping tool, for example, based on single-base primer extension technology, ${ }^{10}$ should be developed. The application of such new methods may remove some markers, because they are located at recurrently mutated loci, mononucleotide repeats or multicopy loci. Finally, the phylogenetic resolving power of the Y-chromosome haplogroup tree presented in their study ${ }^{6}$ will be enhanced by the incorporation of recently upgraded trees. ${ }^{4,5}$

1 Oppenheimer, S. Out-of-Africa, the peopling of continents and islands: tracing uniparental gene trees across the map. Philos. Trans. R. Soc. Lond. B. Biol. Sci 367, 770-784 (2012).

2 Kayser, M. \& de Knijff, P. Improving human forensics through advances in genetics, genomics and molecular biology. Nat. Rev. Genet. 12, 179-192 (2011).

3 Karafet, T. M., Mendez, F. L., Meilerman, M. B., Underhill, P. A., Zegura, S. L. \& Hammer, M. F. New binary polymorphisms reshape and increase resolution of the human Y chromosomal haplogroup tree. Genome Res. 18, 830-838 (2008).

4 Yan, S., Wang, C. C., Li, H., Li, S. L. \& Jin, L. Genographic Consortium. An updated tree of Y-chromosome haplogroup $\mathrm{O}$ and revised phylogenetic positions of mutations P164 and PK4. Eur. J. Hum. Genet. 19, 1013-1015 (2011).

5 Malyarchuk, B. A., Derenko, M. \& Denisova, G. On the Y-chromosome haplogroup C3c classification. J. Hum. Genet. 57, 685-686 (2012).

6 Naitoh, S., Kasahara-Nonaka, I., Minaguchi, K. \& Nambiar, P. Assignment of Y-chromosomal SNPs found in Japanese population to Y-chromosomal haplogroup tree. J. Hum. Genet. 58, 195-201 (2013).

7 Nonaka, I., Minaguchi, K. \& Takezaki, N. Y-chromosomal binary haplogroups in the Japanese population and their relationship to 16 Y-STR polymorphisms. Ann. Hum. Genet. 71, 480-495 (2007).

8 Hammer, M. F., Karafet, T. M., Park, H., Omoto, K., Harihara, S., Stoneking, M. et al. Dual origins of the Japanese: common ground for hunter-gatherer and farmer Y chromosomes. J. Hum. Genet. 51, 47-58 (2006).

9 Kim, S. H., Kim, K. C., Shin, D. J., Jin, H. J., Kwak, K. D., Han, M. S. et al. High frequencies of Y-chromosome haplogroup 02b-SRY465 lineages in Korea: a genetic perspective on the peopling of Korea. Invest. Genet. 2, 10 (2011).

10 van Oven, M., van den Tempel, N. \& Kayser, M. A multiplex SNP assay for the dissection of human $Y$ chromosome haplogroup $O$ representing the major paternal lineage in East and Southeast Asia. J. Hum. Genet. 57, 65-69 (2012). 